Cite this: Chem. Commun., 2014 , 50, 1875

Received 20th November 2013, Accepted 24th December 2013

DOI: $10.1039 / \mathrm{c3cc} 48860 \mathrm{~h}$

www.rsc.org/chemcomm

\section{Modular automated solid phase synthesis of dermatan sulfate oligosaccharides $\dagger$}

\author{
Jeyakumar Kandasamy, ${ }^{a}$ Frank Schuhmacher, ${ }^{a b}$ Heung Sik Hahm, ${ }^{\text {ab }}$ James C. Klein ${ }^{a}$ \\ and Peter H. Seeberger*ab
}

Dermatan sulfates are glycosaminoglycan polysaccharides that serve a multitude of biological roles as part of the extracellular matrix. Orthogonally protected $\mathrm{D}$-galactosamine and L-iduronic acid building blocks and a photo-cleavable linker are instrumental for the automated synthesis of dermatan sulfate oligosaccharides. Conjugationready oligosaccharides were obtained in good yield.

Dermatan sulfate is a glycosaminoglycan (GAG) predominantly found in skin and present in many mammalian tissues. ${ }^{1}$ It is composed of disaccharide repeating units consisting of $N$-acetyl-Dgalactosamine (GalNAc) and L-iduronic acid (IdoA). These alternating disaccharide units can be variably $O$-sulfated at the C-4 and C-6 positions in GalNAc, and the C-2 position in IdoA. ${ }^{2}$ Depending on the source, $63-97 \%$ of the dermatan sulfate polymer is made up of IdoA $\rightarrow$ GalNAc4SO $_{3}$ disaccharide repeating units that are considered to be characteristic of dermatan sulfate (Fig. 1). ${ }^{3}$

Dermatan sulfate binds to a variety of proteoglycans and modulates various biological processes such as coagulation, angiogenesis, tumor migration and growth factor expression. ${ }^{4,5}$ Specific biological roles of dermatan sulfate are poorly understood due to the variations and heterogeneity in its polymer structure. ${ }^{6,7}$ In order to establish structure-activity relationships for dermatan sulfate sequences, to correlate specific sequences and sulfation patterns with protein binding and specific biological activities access to structurally defined dermatan sulfate oligosaccharides is required. Homogeneous samples of such fragments are accessible only via chemical synthesis.

To date, syntheses of dermatan sulfate oligosaccharides have been executed in solution phase relying on many manual operations. ${ }^{8-12}$ With appropriate building blocks in hand, automated solid phase

\footnotetext{
${ }^{a}$ Department of Biomolecular Systems, Max-Planck-Institute of Colloids and Interfaces, Am Mühlenberg 1, 14476 Potsdam-Golm, Germany.

E-mail: peter.seeberger@mpikg.mpg.de; Fax: +49 30 838-59302; Tel: $+4930838-59301$

${ }^{b}$ Freie Universität Berlin, Institute of Chemistry and Biochemistry, Arnimallee 22, 14195 Berlin, Germany

$\dagger$ Electronic supplementary information (ESI) available. See DOI: 10.1039/ c3cc48860h
}

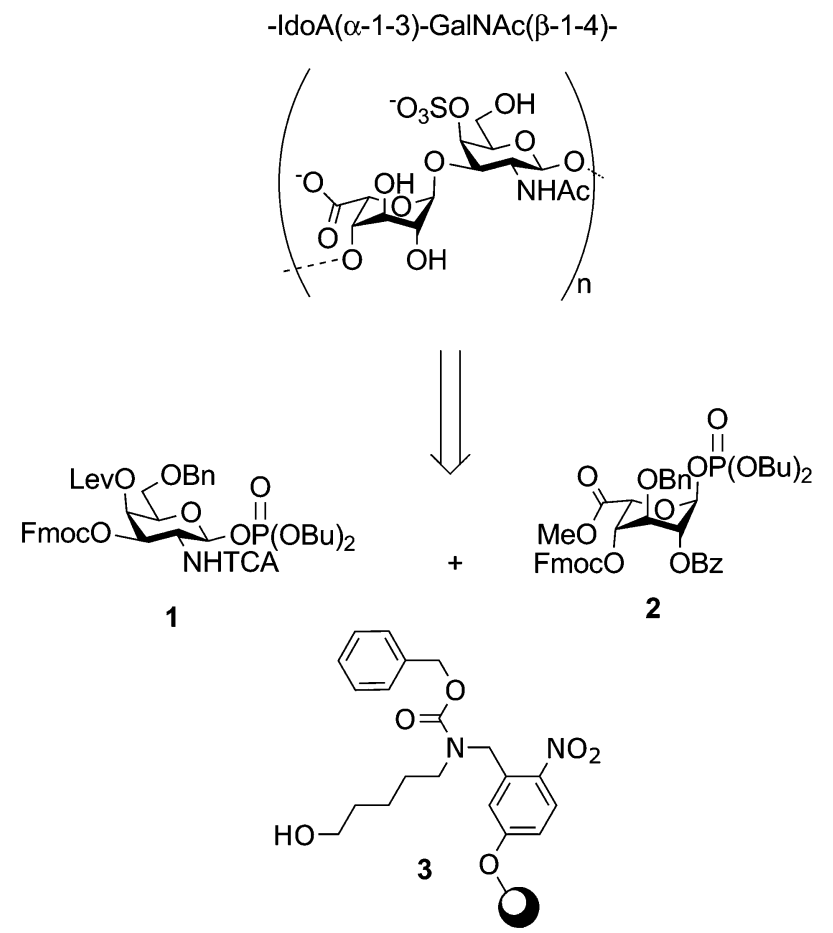

Fig. 1 Structure of dermatan sulfate oligosaccharides and retrosynthetic analysis.

synthesis enables rapid access to structurally-defined oligosaccharides. ${ }^{13,14}$ Automated protocols for the synthesis of other glycosaminoglycans such as hyaluronan ${ }^{15}$ and chondroitin sulfate have been reported previously. ${ }^{16}$ Here, we describe the automated solid phase synthesis of conjugation ready dermatan sulfate oligosaccharides aimed at developing additional tools for biological studies of GAGs.

The dermatan sulfate backbone may arise via assembly of orthogonally protected galactosamine (GalN) and iduronic acid building blocks (1 and 2, Fig. 1) designed for automated solid phase synthesis on solid supported photo-cleavable linker 3 . $^{16}$ The C-3 position of GalN and the C- 4 position of IdoA are masked 

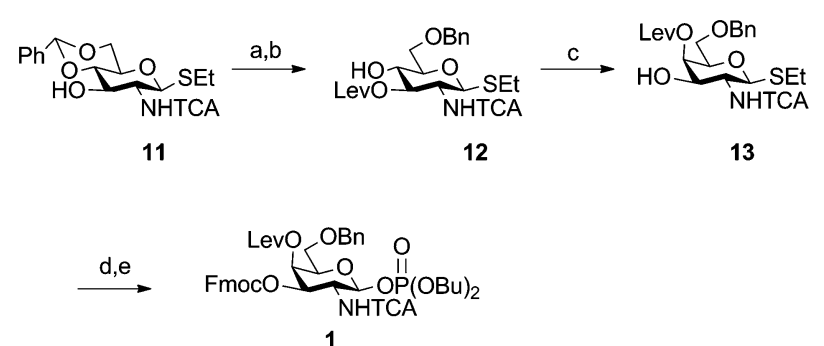

Scheme 1 Synthesis of galactosamine building block 1. Reagents and conditions: (a) Lev $2 \mathrm{O}, \mathrm{Py}, 91 \%$. (b) $\mathrm{Et}_{3} \mathrm{SiH}, \mathrm{TFA}, \mathrm{MS}(4 \mathrm{~A}), 77 \%$. (c) $\mathrm{Tf}_{2} \mathrm{O}, \mathrm{Py},-10{ }^{\circ} \mathrm{C}$, 15 minutes; addition of $\mathrm{H}_{2} \mathrm{O}$, reflux at $\sim 80{ }^{\circ} \mathrm{C}$ for $5 \mathrm{~h}, 78 \%$. (d) $\mathrm{FmocCl}, \mathrm{Py}, 93 \%$. (e) Dibutyl hydrogen phosphate, NIS-TfOH, MS (4 A) , 93\%.

with the temporary 9-fluorenylmethoxycarbonyl (Fmoc) protecting group in anticipation of the regiospecific elongation of the oligosaccharide chain. The C-4 position of GalN carries a levulinoyl ester (Lev) that can be selectively cleaved under mild conditions to allow for sulfation of the free hydroxyl group. The GalN amino group was protected as trichloroacetamide to ensure good $\beta$-selectivities in glycosylation reactions and allow for the installation of the desired acetamide by the direct reduction at the late stage of the synthesis. The C-2 hydroxyl group of the IdoA building block was protected as a benzoate ester to ensure $\alpha$-selectivity in glycosylation reactions. The remaining hydroxyl groups that were not modified during the assembly were permanently protected as benzyl ethers.

Owing to the high cost of galactosamine, these building blocks are accessed from galactose via formation of galactal followed by azido-selenation $^{16,17}$ or from glucosamine via epimerization of the C-4 position. ${ }^{8,9}$ Taking the latter approach into account, we prepared building block 1 in nine steps from glucosamine via migration of $\alpha$-ester with epimerization as the key step (Scheme 1). ${ }^{18,19}$ Thioglycoside $\mathbf{1 1}$ was synthesized in four steps from glucosamine hydrochloride (see ESI $\dagger$ ). ${ }^{20}$ Levulination of the free hydroxyl group in $\mathbf{1 1}$ followed by regioselective ring opening of benzylidine acetal using triethylsilane/trifluoroacetic acid yielded thioglycoside 12 in $70 \%$ yield over two steps. Migration of the C-3 Lev ester to the C-4 hydroxyl with epimerization was effected by transforming the free hydroxyl group in $\mathbf{1 2}$ to the corresponding triflate to produce galactose thioglycoside $\mathbf{1 3}$ in $\mathbf{7 8 \%}$ yield. Fmoc protection of the free hydroxyl group in $\mathbf{1 3}$ followed by the conversion of thioglycoside to glycosyl phosphate using dibutyl hydrogen phosphate and $\mathrm{N}$-iodosuccinimide-triflic acid yielded differentially protected building block 1 in $86 \%$ yield.

Synthesis of IdoA building block 2 was achieved in five steps from thioglycoside $\mathbf{1 4}^{\mathbf{2 1}}$ (Scheme 2). Tetrakis(triphenylphosphine)palladium(0) was employed for the deprotection of the allyl group in thioglycoside $\mathbf{1 4}$ and the resulting hydroxyl group was protected as a benzoyl ester to obtain thioglycoside 15 in $89 \%$ yield (over two steps). Removal of the naphthyl group in 15 using 2,3-dichloro-5,6dicyano-1,4-benzoquinone (DDQ) followed by Fmoc protection of the resulting alcohol provided thioglycoside 16 in $82 \%$ overall yield. Thioglycoside 16 was then converted to the corresponding phosphate glycoside 2 in $92 \%$ yield.

The simple non-sulfated dermatan oligosaccharides were pursued first in order to test the ability of the automated route

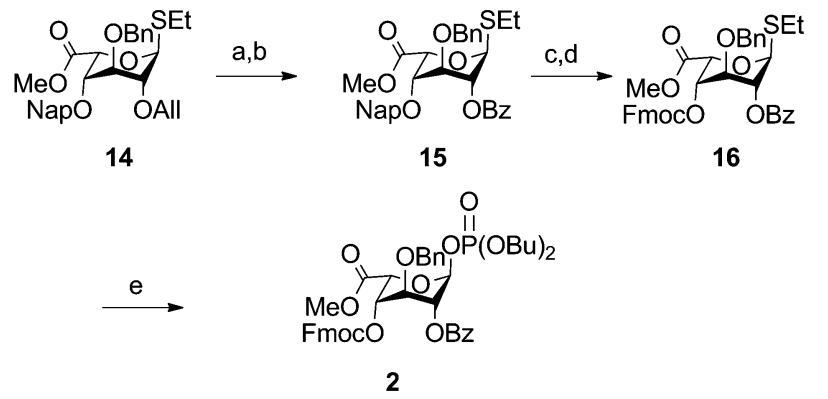

Scheme 2 Synthesis of iduronic acid building block 2 . Reagents and conditions: (a) $\mathrm{Pd}\left(\mathrm{Ph}_{3}\right)_{4}, 1,3$-dimethylbarbituric acid, $\mathrm{MeOH}, 91 \%$. (b) $\mathrm{BzCl}$, Py, 98\%. (c) DDQ, MeOH, 88\%. (d) FmocCl, Py, 94\%. (e) Dibutyl hydrogen phosphate, NIS-TfOH, MS (4 A), 92\%.

to deliver the glycan backbone without deletion sequences. Di- and tetra-saccharides $\mathbf{4}$ and $\mathbf{5}$ were prepared using the oligosaccharide synthesizer via iterative glycosylations with building blocks 1 and 2 and linker 3 (Scheme 3). Glycosylations were carried out at temperatures between $-10{ }^{\circ} \mathrm{C}$ and $0{ }^{\circ} \mathrm{C}$ using trimethylsilyl trifluoromethanesulfonate (TMSOTf). Each cycle involved addition of a glycosylating agent (double coupling) twice, using five equivalents of building blocks. Removal of the Fmoc protecting group with triethylamine uncovered the hydroxyl group ready for elongation.

Synthesis of disaccharide 4 commenced with the first glycosylationdeprotection cycle using building block $\mathbf{1}$ followed by the second glycosylation-deprotection cycle using building block 2 . The resulting free hydroxyl group on the terminal sugar was then acetylated on the synthesizer employing acetic anhydride in pyridine. Disaccharide 4 was released from the resin by applying UV light using a photo flow reactor. ${ }^{16}$ The crude product was purified by HPLC to obtain 4 in $66 \%$ overall yield for six steps based on resin loading. Tetrasaccharide $\mathbf{5}$ was assembled similarly via four glycosylation-deprotection cycles by alternating use of building blocks 1 and 2 , followed by the acetylation of the free hydroxyl group on the terminal sugar. Upon cleavage from the resin, the crude product was purified by HPLC to obtain 6 in $28 \%$ overall yield (ten steps). No deletion sequences were observed in the automated synthesis of $\mathbf{4}$ and $\mathbf{5}$ as judged by HPLC.

Automated synthesis of sulfated dermatan oligosaccharides required two additional steps for installation of sulfates: selective removal of the Lev protecting groups and sulfation of the resulting hydroxyl groups. A solution of hydrazine acetate in pyridine-acetic acid effected removal of the Lev groups at $25{ }^{\circ} \mathrm{C}$. The pyridine sulfur trioxide complex in dimethylformamide-pyridine was used for the sulfation of free hydroxyl groups at $50{ }^{\circ} \mathrm{C}$ on the synthesizer. ${ }^{16}$ Synthesis of sulfated monosaccharide 6 and disaccharide 7 was achieved on a solid support in five and seven automated steps, respectively, under glycosylation-deprotection conditions established for compounds $\mathbf{4}$ and $\mathbf{5}$, followed by automated acetylation, Lev deprotection and sulfation as described above. After the cleavage of the photo-sensitive linker, crude products were analysed by reverse phase (RP)-HPLC and purified by size exclusion chromatography (Sephadex LH-20) to obtain monosaccharide 6 in $43 \%$ and disaccharide 7 in $32 \%$ overall yield based on resin loading.

Automated assembly of sulfated tetrasaccharide $\mathbf{8}$ under the conditions established for the synthesis of 6 and 7 yielded a 


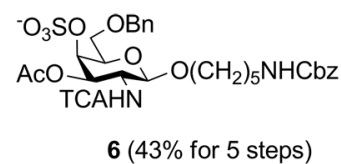
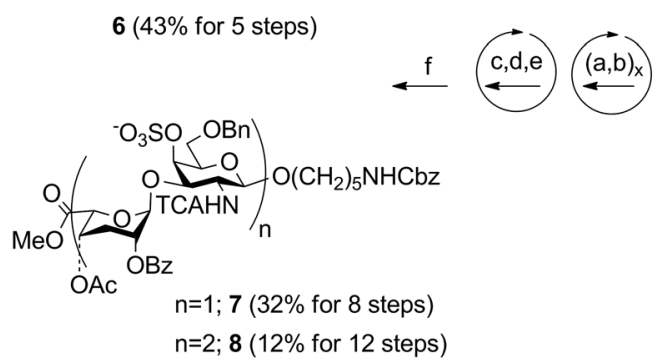

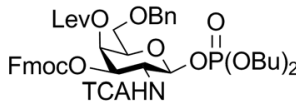

$$
1
$$

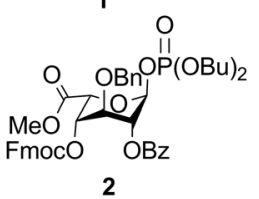

2

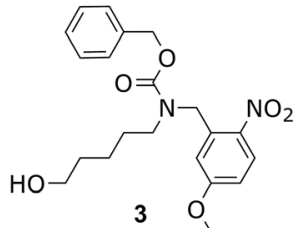

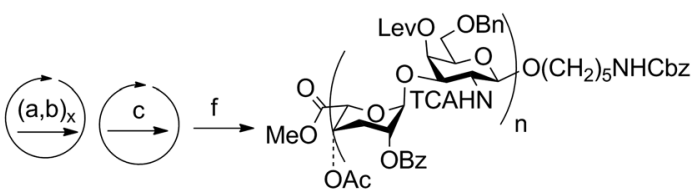

$\mathrm{n}=1 ; 4(66 \%$ for 6 steps $)$ $\mathrm{n}=2 ; \mathbf{5}(28 \%$ for 10 steps $)$

Scheme 3 Automated synthesis of dermatan sulfate oligosaccharide backbones. Reagents and conditions: (a) TMSOTf, DCM, -10 to $0{ }^{\circ} \mathrm{C}$. (b) $25 \% \mathrm{Et}_{3} \mathrm{~N}$ in DMF, $25^{\circ} \mathrm{C}$. (c) $\mathrm{Ac}_{2} \mathrm{O}, \mathrm{Py}, 25^{\circ} \mathrm{C}$. (d) $\mathrm{NH}_{2} \mathrm{NH}_{2} \cdot \mathrm{HOAc}, \mathrm{Py}, \mathrm{AcOH} 25^{\circ} \mathrm{C}$ or $40^{\circ} \mathrm{C}$. (e) Py.SO $\mathrm{SO}_{3}, \mathrm{DMF}, \mathrm{Py}, 50^{\circ} \mathrm{C}$. (f) UV irradiation using continuous flow reactor, $\mathrm{DCM}-\mathrm{MeOH}, \mathrm{RT}$.
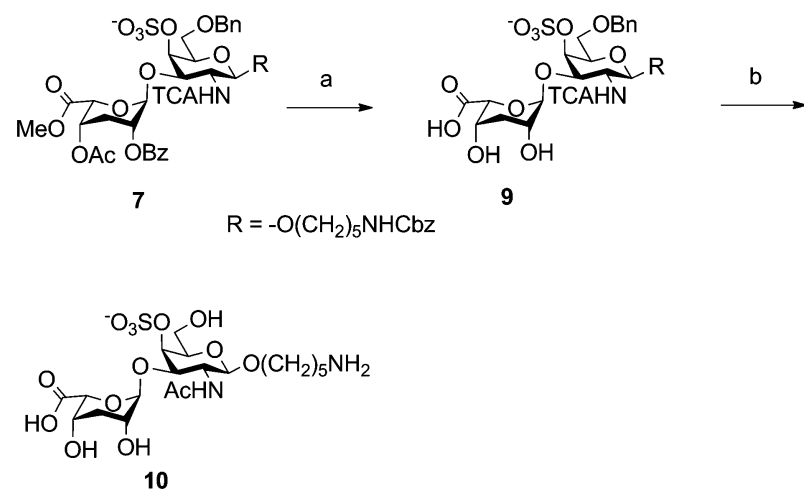

Scheme 4 Deprotection of sulfated disaccharide 7. Reagents and conditions: (a) $1 \mathrm{M} \mathrm{LiOH}, \mathrm{H}_{2} \mathrm{O}_{2}$, THF-MeOH, 79\%. (b) $\mathrm{Pd}-\mathrm{C}, \mathrm{H}_{2}, 80 \%$.

mixture of compounds resulting from incomplete removal of Lev esters with hydrazine acetate at $25{ }^{\circ} \mathrm{C}$. Raising the temperature for Lev deprotection to $40{ }^{\circ} \mathrm{C}$ in the automated run improved the purity and yield of the desired tetrasaccharide 8 that was isolated in $12 \%$ yield over 12 steps.

After assembly of the protected oligosaccharides on the solid support, deprotection of sulfated disaccharide 7 was tested in solution phase (Scheme 4). Hydrolysis of acetate, benzoate and methyl esters was achieved using lithium hydroxide/hydrogen peroxide in THF-MeOH to obtain disaccharide 9 in $79 \%$ yield. Hydrogenolysis of $\mathbf{9}$ provided fully deprotected, conjugation ready disaccharide $\mathbf{1 0}$ in $80 \%$ yield.

In conclusion, an efficient automated solid phase synthesis of dermatan oligosaccharides was achieved using orthogonally protected building blocks and a photo-cleavable linker as well as an automated oligosaccharide synthesizer. Two-step deprotection in solution affords the conjugation ready dermatan sulfate oligosaccharides that can be directly immobilized on microarrays or conjugated to proteins.
We thank the Max-Planck Society and the European Research Council (ERC Advanced Grant AUTOHEPARIN to PHS) for generous financial support. We thank Dr I. Vilotijevic and Dr F. Pfrengle for their help in editing this communication.

\section{Notes and references}

1 J. M. Trowbridge and R. L. Gallo, Glycobiology, 2002, 12, 117R-125R.

2 S. A. Osborne, R. A. Daniel, K. Desilva and R. B. Seymour, Glycobiology, 2008, 18, 225-234.

3 A. M. Halldorsdottir, L. J. Zhang and D. M. Tollefsen, Glycobiology, 2006, 16, 693-701.

4 S. Yamada and K. Sugahara, Curr. Drug Discovery Technol., 2008, 5, 289-301.

5 R. J. Linhardt and R. E. Hileman, Gen. Pharmacol., 1995, 26, 443-451.

6 N. S. Gandhi and R. L. Mancera, Chem. Biol. Drug Des., 2008, 72, 455-482.

7 A. Raghuraman, P. D. Mosier and U. R. Desai, ACS Med. Chem. Lett., 2010, 1, 281-285.

8 N. Barroca and J. C. Jacquinet, Carbohydr. Res., 2000, 329, 667-679.

9 L. Rochepeau-Jobron and J. C. Jacquinet, Carbohydr. Res., 1997, 305, 181-191.

10 J. C. Jacquinet and P. Sinay, Carbohydr. Res., 1987, 159, 229-253.

11 A. Marra, D. Xia, M. Petitou and P. Sinay, Carbohydr. Res., 1989, 195, $39-50$.

12 N. A. Karst and R. J. Linhardt, Curr. Med. Chem., 2003, 10, 1993-2031.

13 O. J. Plante, E. R. Palmacci and P. H. Seeberger, Science, 2001, 291, 1523-1527.

14 M. T. Walvoort, H. van den Elst, O. J. Plante, L. Krock, P. H. Seeberger, H. S. Overkleeft, G. A. van der Marel and J. D. Codee, Angew. Chem., Int. Ed. Engl., 2012, 51, 4393-4396.

15 M. T. Walvoort, A. G. Volbeda, N. R. Reintjens, H. van den Elst, O. J. Plante, H. S. Overkleeft, G. A. van der Marel and J. D. Codee, Org. Lett., 2012, 14, 3776-3779.

16 S. Eller, M. Collot, J. Yin, H. S. Hahm and P. H. Seeberger, Angew. Chem., Int. Ed., 2013, 52, 5858-5861.

17 Y. V. Mironov, A. A. Sherman and N. E. Nifantiev, Tetrahedron Lett., 2004, 45, 9107-9110.

18 R. W. Binkley, J. Org. Chem., 1991, 56, 3892-3896.

19 N. Laurent, D. Lafont, P. Boullanger and J. M. Mallet, Carbohydr. Res., 2005, 340, 1885-1892.

20 L. Krock, D. Esposito, B. Castagner, C. C. Wang, P. Bindschadler and P. H. Seeberger, Chem. Sci., 2012, 3, 1617-1622.

21 P. Bindschadler, A. Adibekian, D. Grunstein and P. H. Seeberger, Carbohydr. Res., 2010, 345, 948-955. 